\title{
Objective assessment of ocular surface as an important prognostic factor for dry eye development in patients with glaucoma.
}

\author{
Anton Vassilev ${ }^{1,2^{*}}$, Violeta Chernodrinska ${ }^{1,2}$, Stanislava Kostova ${ }^{1,2}$, Marta Slavkova ${ }^{3}$, Alexander \\ Oscar $^{1,2}$
}

${ }^{1}$ Department of Ophthalmology, Medical University, Sofia, Bulgaria

${ }^{2}$ Alexandorvska University Hospital, Sofia, Bulgaria

${ }^{3}$ Department of Pharmaceutical Technology and Biopharmacy, Medical University, Sofia, Bulgaria

\begin{abstract}
Objectives: To investigate and compare the tear volume, tear film stability, Meibomian gland loss of healthy and glaucoma subjects from the Bulgarian population and to assess the relationship between clinical findings and glaucoma therapy.

Methods: In this observational cross-sectional study, a random cohort of 68 subjects was enrolled and divided into patients with primary open-angle glaucoma or ocular hypertension (POAG/OHT group; $\mathrm{n}=19$ ) and healthy controls (HC group; $n=49$ ). Ocular surface was analysed objectively by Noninvasive tear Break-Up Time (NIBUT), Meibomian gland loss, and Schirmer test for basal secretion. In addition, Central Corneal Thickness (CCT) was measured with pachymetry.

Results: Loss of Meibomian glands $(39.85 \% \pm 14.49 \%)$, as well as a decrease in Schirmer test values $(7.29 \mathrm{~mm} \pm 3.92 \mathrm{~mm})$ and NIBUT $(8.26 \mathrm{~s} \pm 4.73 \mathrm{~s})$, were typical in the POAG/OHT group, regardless of disease duration. Significant factors affecting the ocular surface state were age, anti-glaucoma treatment (type and duration), and the presence of preservatives. A worsening of all clinical parameters was evident in the glaucoma patients. A correlation between CCT and NIBUT was established ( $p=0.037$; $\mathrm{r}=0.167)$.

Conclusions: Local glaucoma treatment affects the ocular surface objective parameters according to its duration and the composition of treatment eye drops.
\end{abstract}

Keywords: Meibomian gland loss, NIBUT, Dry eye, Central corneal thickness, Schirmer test.

Accepted on July 11, 2018

\section{Introduction}

Dry eye syndrome is a common chronic multifactorial disease that is accompanied by ophthalmic discomfort and changes in visual function [1,2]. Although patients' sensations are the leading cause for seeking medical treatment, very often they cannot be used to assess the ocular surface state, the severity of the condition or the appropriate management approach [3]. Therefore, some objective tests have been used in the practice of dry eye assessment [4]. These can be divided into tests of tear production, tear stability, and surface damage [3]. The Schirmer test is a common invasive technique used to assess tear production; its variations allow complete measurement of both basal and stimulated secretion [5]. Tear stability is characterized via a number of techniques, including different invasive and noninvasive break-up times [6]. Noninvasive Tear Break-Up Time (NIBUT) is a newly developed technique allowing evaluation of the temporal changes in the tear film between the blinks of the patient [7]. The tear film stability is strongly related to the lipid composition and amount in the tear fluid, as this decrease surface tension. These lipids are mainly produced by modified sebaceous glands (Meibomian glands) located on the lower and upper eyelid [3]. Abnormal meibum production can be attributed either to absence or deficiency of Meibomian glands, inflammation, neoplasia, or Meibomian gland dysfunction (e.g, related to hyper- or hyposecretion, or obstruction) [8]. Absence or dysfunction of Meibomian glands is very strongly correlated with dry eye [9].

There is a correlation between anti-glaucoma treatment and the development of dry eye syndrome [10-12]. Current research is mainly focused on the effect that the medications, particularly the preservatives contained herein, may exert on the ocular surface $[10,12]$. The preservatives, especially benzalkonium chloride, are the main factors impairing the normal state and function of the ocular surface [13-15]. Glaucoma represents a heterogeneous group of chronic optic neuropathies, which typically exhibit excavation of the optic nerve head and loss in the visual field [16]. It is considered the primary cause of irreversible blindness worldwide [16]. The most common type 
is the Primary Open-Angle Glaucoma (POAG). Changes in Central Cornea Thickness (CCT) have been associated with an increased risk of damage to the optic nerve head and damage in the visual field [17]. In addition, lower CCT values were observed for patients with dry eye [18].

In the present study, noncontact meibography was performed for the first time in glaucoma and ocular hypertension patients in Bulgaria, in order to evaluate factors leading to the development of dry eye disease. The aim of the study was to objectively characterize and compare the tear film stability and Meibomian gland status in glaucoma patients and healthy volunteers in Bulgaria. It was intended to determine main risk factors regarding more successful and suitable glaucoma treatment and simultaneous prevention of dry eye disease progression.

\section{Materials and Methods}

\section{Patient selection}

The present observational cross-sectional study was in the Department of Ophthalmology at Aleksandrovska University Hospital in Sofia, Bulgaria. The present study was conducted in accordance with the tenets of the Declaration of Helsinki. Patients were divided into two groups, based on the inclusion and exclusion criteria shown in Table 1. The first group were healthy volunteers $(n=49)$ with no history of ocular disease (healthy control (HC) group). The second group consisted of 19 subjects that had been diagnosed with POAG or ocular hypertension (OHT) (POAG/OHT group).

\section{Study protocol}

All patients were subjected to clinical tests of the ocular surface in the following order: NIBUT, Meibography, Schirmer test, and pachymetry, with $5 \mathrm{~min}$ intervals between tests. All measurements were performed first on the right eye, and by a single investigator, to minimize inter-examiner bias.

\section{NIBUT measurement}

The NIBUT was measured using Oculus Keratograph $5 \mathrm{M}^{\circledR}$ (OCULUS, Inc. USA), and recorded in seconds. The evaluation was conducted according to the methodology described by Wiedemann [19]. The procedure was performed in triplicate.

\section{Meibography}

Meibomian gland status was investigated by applying noncontact infrared meibography with Oculus Keratograph $5 \mathrm{M}^{\circledR}$, in accordance with the method suggested by Srinivasan [20]. Local anesthetic (Alcaine ${ }^{\circledR}$, S.A. ALCON, Belgium) was instilled to reduce patient discomfort. Photographs of each eyelid were captured by infrared camera and further analysed by ImageJ 1.50i (Wayne Rasband, National Institutes of Health, Bethesda, MD, USA), as described by Pult and RiedePult [21]. The evaluation was performed objectively based on the percentage of the Meibomian Gland Loss (MGL) on each eyelid. The percentage was calculated according the following formula:

$\operatorname{MGL}(\%)=\mathrm{A}_{\text {drop-out }} / \mathrm{A}_{\text {total }} \times 100 \rightarrow(1)$

where $A_{\text {total }}$ is the total area of the eyelid and the $A_{\text {drop-out }}$ is the area where no Meibomian glands can be seen.

\section{Schirmer test}

The basal tear secretion was measured using Schirmer test ${ }^{\#}$ [5] after the application of topical anaesthesia (Alcaine ${ }^{\circledR}$ ) to reduce reflex secretion caused by conjunctival irritation from the paper strip and to increase patient compliance five minutes after instillation of the eye drops, a $5 \times 35 \mathrm{~mm}$ paper strip (IDEW tear strips, Ophthalmic Experts Ltd., Barking, UK) was folded and gently placed on the temporal one-third of the lower eyelid, bilaterally. During the test, the patient was asked to keep both eyes closed to reduce tear evaporation. The score was recorded as the measured length of wetting from the notch (in $\mathrm{mm}$ ) after five minutes.

\section{Pachymetry}

CCT was measured by Alcon ${ }^{\circledR}$ Ocuscan ${ }^{\circledR}$ RxP pachymeter (Alcon Laboratories, Inc.; Texas; U.S.A) (in $\mu \mathrm{m}$ ). Ten measurements with a standard deviation $<1$ were performed for each eye after applying topical anesthesia (proxymetacaine hydrochloride, Alcaine ${ }^{\mathbb{R}}$ ). The mean values were used for further analysis.

\section{Statistical analysis}

All measured data was subsequently subjected to statistical analysis, including independent samples t-test, ANOVA, Pearson correlation, Spearman correlation, and linear regression using SPSS v. 19.0. A p-value $<0.05$ was regarded as statistically significant.

\section{Results}

\section{Patients}

A total of 68 patients (136 eyes) were included in the study. The age and sex distribution of the patients can be seen in Table 2 .

A statistically significant difference $(\mathrm{p}<0.001 ; \mathrm{df}=134)$ was observed in the mean patient age between the two tested groups.

\section{Schirmer and NIBUT}

In order to determine the influence of sex-related factors, Schirmer test and NIBUT values were statistically analysed with independent samples t-tests to compare each of the tested groups. In the healthy group, a statistically significant difference ( $p=0.003$; df: 88 ) in Schirmer test results was found between men and women. Women exhibit lower values (mean=8.06 $\mathrm{mm} \pm 5.49 \mathrm{~mm}$ ), compared with men (mean=12.33 $\mathrm{mm} \pm 7.69 \mathrm{~mm}$ ). In the HC group, women 


\section{glaucoma}

age $>45$ y showed lower Schirmer values $(7.16 \pm 6.32 \mathrm{~mm}$; $\mathrm{p}=0.015$ ) compared with men at any age. Similar results were found in the POAG/OHT (women age $>45 \mathrm{y}: 6.14 \pm 4.86 \mathrm{~mm}$; men at any age $8.89 \pm 5.73 \mathrm{~mm} ; \mathrm{p}<0.01)$.

The age of the patients in the HC group revealed a significant Pearson correlation with the NIBUT values $(p<0.037$; $\mathrm{r}=-0.214)$. A stronger correlation between the same parameters was found in the POAG/OHT group ( $\mathrm{p}=0.018$; $\mathrm{r}=-0.381)$. In addition, a decrease in the volume and tear film stability was established between the HC group and the POAG/OHT group as can be seen in Figure 1A.

It can be noticed that there is a very strong correlation between NIBUT values and age of the patients in the POAG/OHT groups when patients are divided by gender $(\mathrm{p}=0.005$; $\mathrm{r}=-0.750)$. Other age and gender related differences were not established.

\section{Meibomian glands}

The investigation of the Meibomian glands status in the tested groups was based on analysis of images obtained by the Oculus Keratograph 5M. The total area and the area without Meibomian glands can be seen on the Figure 2.

There was statistical differences between MGL measurements in the POAG/OHT and HC groups for both lower ( $\mathrm{p}=0.029$; $\mathrm{df}$ : 134) and upper eyelid $(\mathrm{p}<0.001$, df: 134). The results are shown in Figure 1B. Approximately $10 \%$ greater total gland loss is detected in the POAG/OHT group $(39.85 \% \pm 14.49 \%)$, compared with the healthy group $(27.10 \% \pm 13.98 \%)$.

For the HC group, age was correlated with MGL on the upper eyelid $(\mathrm{r}=0.271 ; \mathrm{p}=0.007)$. In the POAG/OHT group, a significant correlation was found only for the lower eyelid (LL) $(\mathrm{r}=0.331 ; \mathrm{p}=0.042)$. The total MGL was correlated with age $(r=0.219 ; \mathrm{p}=0.031)$ and NIBUT $(\mathrm{p}=0.003 ; \mathrm{r}=-0.303)$ in the healthy group. An independent sample t-test showed that, in the $\mathrm{HC}$ group, women age $>45$ y exhibited a statistically significant decrease in the MGL-LL $(26.15 \pm 14.61 ; \mathrm{p}<0.01)$, compare with younger women $(14.59 \pm 8.91 \%)$. As expected, no correlation of Schirmer test results with MGL was established in either of the groups.

\section{$C C T$}

The CCT pachymetry results showed some significant differences between the HC and POAG/OHT group $(\mathrm{p}<0.001)$. The mean CCT in the healthy cohort was $569.0 \pm 30.4 \mu \mathrm{m}$ whereas in the POAG/OHT group it was $535.9 \pm 31.5 \mu \mathrm{m}$. In the POAG/OHT group approximately CCT values were $30 \mu \mathrm{m}$ lower than those in the $\mathrm{HC}$ group. Further investigation of the results showed that there was a correlation between the parameter characterizing tear film stability and the CCT in the POAG/OHT group $(\mathrm{p}=0.037 ; \mathrm{r}=0.167)$. A regression analysis with confidence interval of $95 \%$ was performed.

A correlation was found between the CCT and the patients' age in the POAG/OHT group $(\mathrm{p}=0.024 ; \mathrm{r}=-0.386)$.

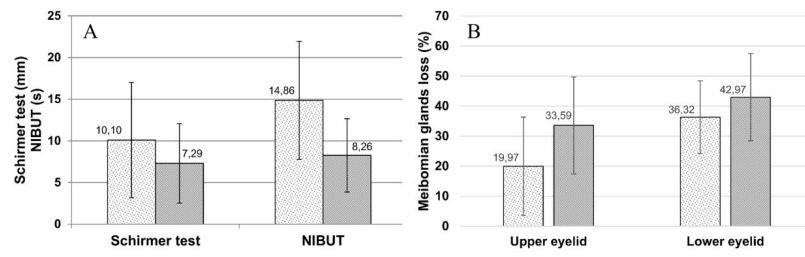

Figure 1. A: Mean values $\pm S D$ of the estimated Schirmer test (in mm; $p<0.032$ ) and NIBUT (in $s ; p<0.001$ ); B: Meibomian gland loss on upper and lower eyelid presented as \% of the total eyelid area for the tested groups $\pm S D$ (HG group; POAG/OHT group).

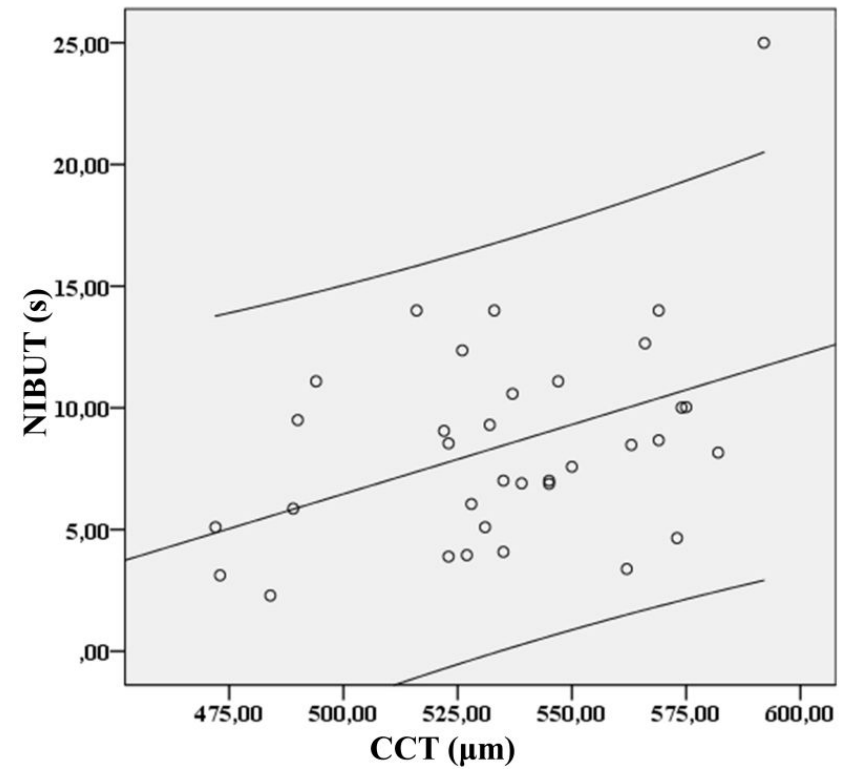

Figure 2. Evaluation of the area in pixels using Image $J$ (A-total area of the upper eyelid marked within the line; $B$ - area of the upper eyelid without Meibomian glands within the line).

\section{Correlation analysis}

Pearson correlation analysis was conducted. Some differences were present between the $\mathrm{HC}$ and POAG/OHT groups (Table $3)$.

\section{Influence of glaucoma treatment}

The influence of treatment duration on the main tested parameters in the POAG/OHT group was statistically investigated. The results are shown in Table 4.

NIBUT was affected significantly by the duration of treatment and number of Active Pharmaceutical Ingredients (APIs): longer duration and a greater number of drugs were associated with lower NIBUT values. The number of APIs simply shows the number of active substances of different therapeutic groups is used: it is not related to the number of commercial products, because some API combinations exist as eye drops on the market. CCT decreased with increased treatment duration, although the effect was not statistically significant. No correlation was established between the MGL on the lower or upper eyelid with continuous therapy, and with an increased number of APIs. The type of API significantly affected MGL- 
LL when beta-blockers were applied. The Schirmer test results were influenced by the API type in cases where carbonic anhydrase inhibitors were used. A significant presence of Benzalkonium Chloride (BAC) was established for the eye drops containing beta-blockers $(\mathrm{p}=0.001)$ or Carbonic Anhydrase Inhibitors (CAI) $(\mathrm{p}=0.045)$. The presence of preservative (BAC) in the eye drops showed a significant effect on MGL on the lower eyelid ( $p=0.039$; $r=-0.336)$.

Table 1. Inclusion/exclusion criteria for study participants (POAG-Primary Open Angle Glaucoma; OHT: Ocular Hypertension; HG: Healthy Group).

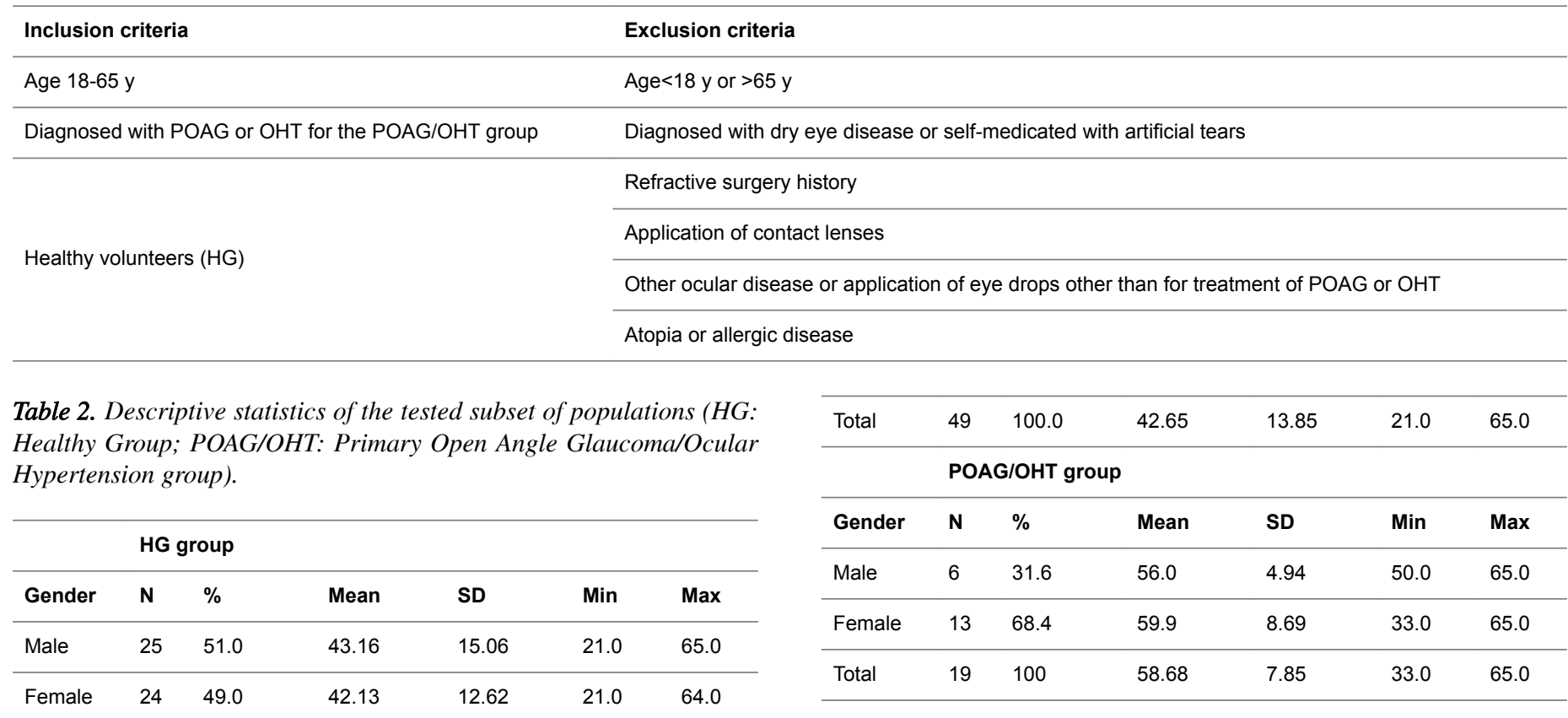

Table 3. Pearson correlation analysis in the POAG/OHT group (MGL-UL Meibomian Gland Loss-Upper Eyelid; MGL-LL Meibomian Glands Loss-Lower Eyelid) (significant correlations are marked in red).

\begin{tabular}{|c|c|c|c|c|c|c|c|c|}
\hline \multirow{2}{*}{ Parameter } & \multicolumn{2}{|c|}{ MGL-LL (\%) } & \multicolumn{2}{|c|}{ NIBUT (s) } & \multicolumn{2}{|c|}{ Schirmer (mm) } & \multicolumn{2}{|l|}{$\mathrm{CCT}(\mu \mathrm{m})$} \\
\hline & HG & POAG/OHT & HG & POAG/OHT & HG & POAG/OHT & HG & POAG/OHT \\
\hline \multirow{2}{*}{ MGL-UL (\%) } & $r=0.427$ & $r=0.130$ & $r=-0.181$ & $r=-0.423$ & $r=0.146$ & $r=-0.128$ & $r=-0.085$ & $r=-0.193$ \\
\hline & $p<0.001$ & $p=0.436$ & $p=0.077$ & $p=0.008$ & $p=0.169$ & $p=0.472$ & $p=0.692$ & $p=0.247$ \\
\hline \multirow{2}{*}{ MGL-LL (\%) } & $r=1$ & $r=1$ & $r=-0.327$ & $r=-0.034$ & $r=0.025$ & $r=-0.108$ & $r=-0.029$ & $r=-0.032$ \\
\hline & & & $p=0.001$ & $p=0.838$ & $p=0.818$ & $p=0.545$ & $p=0.893$ & $p=0.857$ \\
\hline \multirow{2}{*}{ NIBUT (s) } & & & $r=1$ & $r=1$ & $r=0.307$ & $r=0.080$ & $r=-0.151$ & $r=0.358$ \\
\hline & & & & & $p=0.003$ & $p=0.653$ & $p=0.481$ & $p=0.037$ \\
\hline \multirow{2}{*}{ Schirmer (mm) } & & & & & $r=1$ & $r=1$ & $r=0.083$ & $r=-0.135$ \\
\hline & & & & & & & $p=0.698$ & $p=0.475$ \\
\hline
\end{tabular}

Table 4. Correlation coefficients and p-values for the main tested parameters and treatment characteristics (API: Active Pharmaceutical Ingredient; BAC: Benzalkonium Chloride; CAI: Carboanhydrase Inhibitor).

\begin{tabular}{lllc}
\hline Investigated parameter & Treatment duration & Number of API & Type of API \\
\hline MGL-UL (\%) & $r=-0.193$ & $r=-0.042$ & $p>0.05$ \\
\cline { 2 - 4 } & $p=0.247$ & $p=0.803$ & $r=0.168$ \\
\hline MGL-LL (\%) & $r=-0.211$ & $r=-0.231$ & $r=-0.373$ \\
\cline { 2 - 4 } & $p=0.205$ & $p=0.163$ & $p=0.021(\beta-b l o c k e r)$ \\
\hline
\end{tabular}




\begin{tabular}{|c|c|c|c|c|}
\hline \multirow{2}{*}{ Schirmer (mm) } & $r=0.107$ & $r=-0.138$ & $r=-0.483$ & $r=0.030$ \\
\hline & $p=0.546$ & $p=0.436$ & $\mathrm{p}=0.004(\mathrm{CAl})$ & $p=0.865$ \\
\hline \multirow{2}{*}{ NIBUT (s) } & $r=-0.334$ & $r=-0.334$ & $p>0.05$ & $r=0.087$ \\
\hline & $\mathrm{p}=0.027$ & $p=0.040$ & & $p=0.605$ \\
\hline \multirow{2}{*}{$\mathrm{CCT}(\mu \mathrm{m})$} & $p=-0.334$ & $r=-0.156$ & $p>0.05$ & $r=0.027$ \\
\hline & $p=0.053$ & $p=0.378$ & & $p=0.879$ \\
\hline
\end{tabular}

\section{Discussion}

There are few other studies investigating dry eye disease by simultaneously using MGL, NIBUT and Schirmer test for basal secretion in dry eye patients $[7,8,10,19,20]$. According to the available literature, there are no other studies using all of these objective tests for dry eye assessment in glaucoma patients in Bulgaria. The subjects in the present study were selected based on a wide age range, as it was necessary to determine if the changes in the clinical findings were related to patient age. The upper limit (65 y) was selected following the World Health Organization definition of elderly people [22]. The elderly are usually co-morbid and some of the changes in their ocular status could be a result of a variety of systemic diseases. Therefore, they were not included in the study. In addition, there is data showing that $15 \%$ of the people older than 65 y suffer from dry eye disease [23]. Furthermore, the patients included in the POAG/OHT group were either newly diagnosed or have been treated for glaucoma for many years.

A difference was found concerning the mean age in the two groups. The main reason for the higher age of the patients from $\mathrm{POAG} / \mathrm{OHT}$ group, compared with the $\mathrm{HC}$ group, is that glaucoma and ocular hypertension are age-related diseases and prevail in patients older than $45 \mathrm{y}$ [24]. This age difference could lead to some bias in the data comparison between the two groups. The upper limit set in our exclusion criteria helped to decrease the influence of age on the results. Furthermore, it is necessary to include patients with various treatment durations regarding the influence of therapy on POAG/OHT. The prevalence of women in the POAG/OHT group might be explained by an increased willingness for evaluation.

Dry eye disease is predominantly associated with women [25]. The women in our HC group were characterized with lower Schirmer test values than the men in the same group. This might be a result of the sex-related differences in the lacrimal glands $[25,26]$. In the present study, no significant difference in tear secretion was found between men and women in the POAG/OHT group. However, it was evident that women age $>45$ y presented lower Schirmer test values than younger women or men at any age. This could be explained by hormonal changes that occur after menopause.

The tear film stability evaluated by the NIBUT was shown to be age-dependent; however, concerning the sex there was no relation in any of the groups. Similar results have been established by other investigators as well [27]. Therefore, sex can be eliminated as a risk factor in patients with glaucoma. In this subpopulation other characteristics are responsible for dry eye development.

By comparing the tested groups it was evident that the quantity and quality of tear film in the POAG/OHT group is lower than the healthy group. Our results undoubtedly show a relation between glaucoma and dry eye. Such a concept has been proposed by many authors [11,28,29]. The exact pathophysiological mechanism behind this relationship remains unclear [29]. The results obtained in this study regarding tear film volume and stability, show significant decreases in the values for the POAG/OHT group. Thus, the evaporative and aqueous deficient components of dry eye can be considered to be present in patients with glaucoma. Likely, this is induced by Meibomian gland deficiency, as well as desiccating stress on the ocular surface. This theory has been suggested by other researchers $[28,30]$.

The total MGL was significantly different in the two test groups, showing that patients with glaucoma are characterized by higher MGL. This MGL is not age-related, as no significant correlation was established between the total MGL and patient age in the POAG/OHT group. Other factors may affect the loss of Meibomian glands, such as environmental stress and topical medications [31]. The significant difference of MGL on the lower eyelid between women age $>45 \mathrm{y}$ and younger women may be due to hormonal changes that occur after menopause. However, such a difference is found only for the lower eyelid in the HC group. Therefore, it may be that, in patients with glaucoma, the hormonal status does not influence the ocular state.

The Spearman correlation in this study showed that the type of API and the presence of BAC are related to the loss of Meibomian glands on the lower eyelid. This might be related to the manner of instillation of eye drops, as the first contact is directly with the lower eyelid. MGL on the upper eyelid is better correlated to the stability of the tear film, in contrast with MGL on the lower eyelid. Similar results have been reported by Pult et al. [32].

Topical glaucoma treatment has a significant versatile effect on the ocular surface. The application of $\beta$-blockers induces Meibomian gland alterations. The instillation of CAIs affects basal tear production. Thus, long-term treatment contributes to a decrease in NIBUT. In the present study, BAC-containing eye drops had a significant effect on the Meibomian glands. Our results partially confirm previously reported data [33]. Glaucoma therapy seems to be one of the most critical factors 
influencing the surface state in dry eye. This highlights the complexity of the problem and shows that the clinical findings continue to be inconsistent between studies [34]. It is, therefore, very important to choose suitable anti-glaucoma therapies with regard to the state of the ocular surface.

Additionally parameters typically tested for glaucoma include CCT, were evaluated in relation to ocular surface parameters. In the present study, a correlation was found between CCT and the NIBUT in the POAG/OHT group; however, a similar observation was not present for the HC group. A previous study [26] reported a correlation between dry eye disease and thinner central cornea in women. Nevertheless, a correlation between CCT and NIBUT has not yet been reported; further investigation is needed to determine the relationship between corneal thicknesses and tear film stability. Notably, low CCT values are associated with an increased risk of glaucoma. The results in the present study how a potential for CCT measurements to identify the risk of dry eye development in earlier stages, before clinical signs are evident.

\section{Conclusion}

This study showed a relationship between POAG or OHT and the development of dry eye. A worsening in the ocular surface status was observed for the patients with glaucoma, compared with healthy volunteers. Many factors are important in the damage of the ocular surface, including age, sex, and glaucoma treatment. Interestingly, a correlation between CCT and tear film stability was established in the present study. The low CCT can be considered an additional prognostic tool for the early detection of dry eye. Importantly, treatment of glaucoma can affect the ocular surface because of its duration, complexity, and the presence of preservatives: it influences a variety of clinical parameters, such as MGL-LL, Schirmer test, and NIBUT.

\section{References}

1. Craig JP, Nichols KK, Akpek EK, Caffery B, Dua HS, Joo CK, Liu Z, Nelson JD, Nichols JJ, Tsubota K, Stapleton F. TFOS DEWS II definition and classification report. Ocul Surf 2017; 15: 276-283.

2. Tsubota K, Yokoi N, Shimazaki J, Watanabe H, Dogru M, Yamada M, Kinoshita S, Kim HM, Tchah HW, Hyon JY, Yoon KC, Seo KY, Sun X, Chen W, Liang L, Li M, Liu Z, Asia Dry Eye Society. New perspectives on dry eye definition and diagnosis: a consensus report by the Asia dry eye society. Ocul Surf 2017; 15: 65-76.

3. McGinnigle S, Naroo SA, Eperjesi F. Evaluation of dry eye. Surv Ophthalmol 2012; 57: 293-316.

4. Wolffsohn JS, Arita R, Chalmers R, Djalilian A, Dogru M, Dumbleton K, Gupta PK, Karpecki P, Lazreg S, Pult H, Sullivan BD, Tomlinson A, Tong L, Villani E, Yoon $\mathrm{KC}$, Jones L, Craig JP. TFOS DEWS II diagnostic methodology report. Ocul Surf 2017; 15: 539-574.
5. Bitton E, Wittich W. Influence of eye position on the Schirmer tear test. Cont Lens Anterior Eye 2014; 37: 257-261.

6. Sweeney DF, Millar TJ, Raju SR. Tear film stability: a review. Exp Eye Res 2013; 117: 28-38.

7. Hong J, Sun X, Wei A, Cui X, Li Y, Qian T, Wang W, Xu J. Assessment of tear film stability in dry eye with a newly developed keratograph. Cornea 2013; 32: 716-721.

8. Suzuki T, Teramukai S, Kinoshita S. Meibomian glands and ocular surface inflammation. Ocul Surf 2015; 13: 133-149.

9. Lee KW, Kim JYC. Assessment of the tear meniscus by strip meniscometry and keratograph in patients with dry eye disease according to the presence of meibomian gland dysfunction. Cornea 2017; 36: 189-195.

10. Saini M, Vanathi M, Dada T, Agarwal T, Dhiman R, Khokhar S. Ocular surface evaluation in eyes with chronic glaucoma on long term topical antiglaucoma therapy. Int $\mathrm{J}$ Ophthalmol 2017; 10: 931-938.

11. Yuksel N. Evaluation of ocular surface disease associated with glaucoma patients. Eur Ophthal Rev 2013; 7: 81-83.

12. Aptel F, Labbe A, Baudouin C. Treatment of glaucoma, preservatives and ocular surface. J Francais Ophthalmol 2014; 37: 728-736.

13. Baudouin $\mathrm{C}$, Traverso CE. The role of preservative-free therapies in the treatment of glaucoma. Eur Ophthal Rev 2013; 7: 74-80.

14. Noecker R, Miller KV. Benzalkonium chloride in glaucoma medications. Ocul Surf 2011; 9: 159-162.

15. Iester M, Telani S, Frezzotti P. Ocular surface changes in glaucomatous patients treated with and without preservatives beta-blockers. J Ocul Pharmacol Ther 2014; 1-6.

16. Jonas JB, Aung T, Bourne RR, Bron AM, Ritch R, PandaJonas S. Glaucoma. Lancet 2017; 390: 2183-2193.

17. Liu B, McNally S, Kilpatrick JI, Jarvis SP, OBrien CJ. Aging and ocular tissue stiffness in glaucoma. Surv Ophthalmol 2018; 63: 56-74.

18. Akdemir MO, Kirgiz A, Ayar O, Kaldirim H, Mert M, Cabuk KS, Taskapili M. The effect of pseudoexfoliation and pseudoexfoliation induced dry eye on central corneal thickness. Curr Eye Res 2016; 41: 305-310.

19. Wiedemann D, Sickenberger W, Michel M. Development and realization of a new non-invasive method for tear film assessment using a corneal topographer. Poster Session presented at American Academy of Optometry, San Francisco 2010.

20. Srinivasan S, Menzies K, Sorbara L, Jones L. Infrared imaging of meibomian gland structure using a novel keratograph. Optom Vis Sci 2012; 89: 788-794.

21. Pult H, Riede-Pult BH. Non-contact meibography: keep it simple but effective. Cont Lens Anterior Eye 2012; 35: 77-80.

22. WHO. Definition of older and elderly person. Internet Geneva, Switzerland: WHO 2010. 


\section{glaucoma}

23. Moss SE, Klein R, Klein BE. Incidence of dry eye in an older population. Arch Ophthalmol 2004; 122: 369-373.

24. Moss SE, Klein R, Klein BE. Prevalence of and risk factors for dry eye syndrome. Arch Ophthalmol 2000; 118: 1264-1268.

25. Sullivan DA, Rocha EM, Aragona P, Clayton JA, Ding J, Golebiowski B, Hampel U, McDermott AM, Schaumberg DA, Srinivasan S, Versura P, Willcox MDP. TFOS DEWS II Sex, Gender, and Hormones Report. Ocul Surf 2017; 15: 284-333.

26. Wagner H, Fink BA, Zadnik K. Sex- and gender-based differences in healthy and diseased eyes. Optometry 2008; 79: 636-652.

27. Sharanjeet K, Ho CY, Mutalib HA, Ghazali AR. The relationship between tear ferning patterns and noninvasive tear break-up time in normal Asian population. J Optom 2016; 9: 175-181.

28. Gomes JAP, Azar DT, Baudouin C, Efron N, Hirayama M, Horwath-Winter J, Kim T, Mehta JS, Messmer EM, Pepose JS, Sangwan VS, Weiner AL, Wilson SE, Wolffsohn JS. TFOS DEWS II iatrogenic report. Ocul Surf 2017; 15: 511-538.

29. Steven P, Cursiefen C. Glaucoma and dry eye. Current concepts and future perspectives. Ophthalmologe 2013; 110: 1155-1159.
30. Pflugfelder SC, Stern M, Zhang S, Shojaei A. LFA-1/ ICAM-1 interaction as a therapeutic target in dry eye disease. J Ocul Pharmacol Ther 2017; 33: 5-12.

31. Chhadva P, Goldhardt R, Galor A. Meibomian gland disease: the role of gland dysfunction in dry eye disease. Ophthalmology 2017; 124: 20-26.

32. Pult H, Riede-Pult BH, Nichols JJ. Relation between upper and lower lids Meibomian gland morphology, tear film, and dry eye. Optom Vis Sci 2012; 89: 310-315.

33. Arita R, Itoh K, Maeda S. Comparison of the long-term effects of various topical antiglaucoma medications on Meibomian glands. Cornea 2012; 31: 1229-1234.

34. Tressler CS, Beatty R, Lemp MA. Preservative use in topical glaucoma medications. Ocul Surf 2011; 9: 140-158.

\section{*Correspondence to}

Anton Vassilev

Department of Ophthalmology

Medical University

Bulgaria 